\title{
Melody and Language: An Examination of the Relationship Between Complementary Processes
}

\author{
Victoria L. Harms, Colleen Cochran, and Lorin J. Elias*
}

Department of Psychology, University of Saskatchewan, Canada

\begin{abstract}
It is well accepted that the left and right hemispheres of the brain typically play separate and distinct roles in cognitive processing. Extensive research examining the lateralization of music and language processes has provided a clear and consistent demonstration of this division of processing across the cerebral hemispheres. However, in spite of this line of research examining population-level lateralization of these processes, little focus has been placed on examining the relationship between the two processes. Do these two processes share a common developmental origin that influences their pattern of lateralization, or do independent processes govern their lateralization? In this study we examined the relationship pattern in degree of lateralization between linguistic processing and melody recognition using dichoticlistening tasks. The expected right ear advantage was observed for the linguistic processing task. Additionally, the expected left ear advantage was not observed for the melody recognition task, precluding an informative assessment of complementarity between the two tasks. A positive correlation between the laterality scores on the two tasks suggests a shared processing network for linguistic and melodic processing.
\end{abstract}

Keywords: Lateralization, complementarity, language, music, melody recognition.

Music and language are both unique in that they are exclusive to human experience and they are both universally present in every culture [1]. In addition, both functions share common processing features - music and language both rely on the ability to process temporal auditory complexity, tonal structure at the global and local level, syntax, and sequence [2]. Indeed, there is some strong evidence to suggest a large degree of overlap in the processing networks for music and language [3-6]. Finally, both functions are known to be highly lateralized, with specific features of music and language processing showing left or right hemisphere processing advantages [7]. The degree of similarity in processing and evidence of a shared processing network hints at a common developmental origin underlying these two cognitive functions [8-10]. However, we cannot provide informative and effectively testable theories of developmental origin for these functions until we understand the relationship between them. This invites the question: do these two cognitive functions share a common lateralizing influence?

Music is multifaceted with both the left and right hemispheres playing active roles in the different aspects of musical-stimuli processing. The left hemisphere is involved in rhythm and temporal processing [11], familiarity [12, 13], and local interval processing $[14,15]$. The right hemisphere is known to be involved in contour processing [16], harmony [17], and melody recognition $[18,19]$. Melodic processing is

*Address correspondence to this author at the Department of Psychology, University of Saskatchewan, 9 Campus Drive, Saskatoon, Saskatchewan, S7N 5A5, Canada; Tel: 306-966-6657; Fax: 306-966-6630;

E-mail: lorin.elias@usask.ca information in much the same way as language; a melody can convey meaning through syntactic structure, emotion, emphasis, and context by mirroring or contrasting a particular theme or feel in tempo, pitch pattern, and dynamics [2].

Evidence for right-hemisphere dominance in melodic processing comes from three key sources: first, work focused on patient populations with unilateral lesions or lobectomies has revealed a consistent pattern of melodic recognition deficit following right-hemisphere damage [12, 20-22]. Steinke, Cuddy, and Jakobson [23] examined melody recognition in a stroke patient $(\mathrm{KB})$ with right-hemisphere damage. $\mathrm{KB}$ demonstrated significant impairment in familiar melody recognition for instrumental melodies. Interestingly, $\mathrm{KB}$ was still able to recognize familiar song (lyrical) melodies even when the melodies were played without the lyrics.

A second source of evidence for right-hemisphere dominance in melody recognition comes from dichoticlistening studies in neurologically healthy populations $[18$, 24, 25]. Boucher and Bryden [26] used one-second, singleinstrument melodies excerpted from classical works, which they played at a speeded rate of 400 bps. They presented dichotic pairs of the melodies and asked participants listen for a specific target melody. An overall left ear advantage (LEA) for melody recognition was observed.

The third source of evidence for right-hemisphere dominance in melody recognition comes from neuroimaging work in neurologically healthy populations [27, 28]. Patterson, Uppenkamp, Johnsrude, and Griffiths [29] found 
greater right-hemisphere activation in the temporal region using fMRI in response to melodic stimuli compared to fixed-pitch stimuli. Additional work by Platel et al. [13] examined the influence of familiarity on melody recognition and found right-hemisphere dominance when the melodies were unfamiliar but found more bilateral activation when melodies were familiar to the participants. Taken together, there is clear evidence for a dominant role of the right hemisphere in processing melodic information.

Language, much like music, is also multifaceted, and involves both left and right hemisphere processes. The right hemisphere is shown to be involved in processing the paralinguistic features of language such as prosody [30-34]. However, it is the left hemisphere's dominance for processing semantic meaning, syntax, and phonology that is the hallmark of language lateralization [35-39].

This population-level pattern of lateralization for linguistic and melodic processing is consistent with Bryden's [40] Modal Model of cerebral organization, which proposes a differentiation of processing abilities between the two hemispheres [41,42] resulting in more efficient cognitive processing [43, 44]. According to this view, the right hemisphere is specialized for holistic, integrative processing of information whereas the left hemisphere is specialized for serial, sequential, and analytic processing of information [45]. This complementary organization of left- and rightlateralized functions is often taken as evidence for the Modal Model; however, an examination of the correlation of the degree of lateralization between these functions withinsubjects is necessary to establish whether or not a true relationship is present.

Three possible patterns of complementarity can be observed when examining the relationship between a leftand a right-lateralized cognitive process. The first pattern is causal complementarity [40, 46]. This pattern reflects a relationship where the asymmetrical lateralization of one cognitive function impels the opposite lateralization of the other function. For example, the specialization of the left hemisphere for linguistic processing leads to righthemispheric lateralization for non-linguistic processes. As this pattern predicts opposite lateralization for complementary processes, in cases where language is found to be lateralized to the right hemisphere, this pattern would predict that non-linguistic processes would then be lateralized to the left-hemisphere. As such, a negative correlation in the degree of lateralization between the left- and right-hemisphere functions should be observed.

The second pattern is bias complementarity [40]. This pattern reflects the influence of asymmetries of either ascending sensory systems or attention, producing an overall bias for cognitive processing [47-50]. As the bias influences either a complete sensory system or a global attentional mechanism the bias complementarity pattern predicts that a strong right ear advantage (REA) for a left hemisphere lateralized task will be accompanied by a weak LEA for a right hemisphere lateralized task (and vice versa); thus, a positive correlation in the degree of lateralization between the two functions should be observed.
The third pattern is statistical complementarity [40, 46, 51]. This pattern reflects independent sources influencing asymmetrical lateralization of cognitive processes. As such, each cognitive function carries a specific probability of being lateralized to either the right or left hemisphere depending on the source of influence. The two functions may be consistently lateralized to opposite hemispheres through separate, independent influences. A statistical pattern suggests that the population-level pattern of lateralization has been determined by chance and that the lateralization of one process to a particular hemisphere says nothing of the nature of the processes lateralized to the other hemisphere within an individual [51]. An examination of the relationship between two such statistically independent cognitive functions would reveal no correlation.

Few studies have directly examined the relationship in degree and direction of lateralization between left- and rightlateralized tasks within individuals. Ley and Bryden [32] examined complementarity of linguistic and emotional processing using sentences spoken in emotional tones. They observed the expected population-level pattern with a REA observed for the linguistic content of the sentence stimuli and a LEA for the emotional content; however, when the relationship between the left- and right-lateralized tasks was examined, no significant correlation was observed. The authors argued that this result provides support for the statistical model of complementarity. Using a similar method, Saxby and Bryden [52] assessed the lateralization of emotional and linguistic processing in kindergarten, grade 4, and grade 8 children; they found no predictive relationship in the degree of lateralization for linguistic and emotional processing, again suggesting a statistical pattern of complementarity. Finally, similar evidence in support of statistical complementarity was found by McNeely and Parlow [53] who also examined the complementarity of linguistic and prosodic processing. They measured linguistic lateralization using the Fused Words Dichotic-listening Task (FWDT) and measured prosodic lateralization using the Dichotic Emotion Recognition Test (DERT). The expected population-level pattern was observed: an overall REA for linguistic processing and an overall LEA for prosodic processing, but no significant correlation between the two functions was observed when the relationship was examined within individuals.

Similar evidence in support of statistical complementarity has come from recent studies using functional transcranial Doppler (fTCD) to measure lateral differences in cerebral blood flow. Whitehouse and Bishop [54] examined the relationship between fTCD laterality indices (LI) for both visuospatial memory and lingusitic processing. They found no significant relationship between the two complementary functions. Similarly, Rosch, Bishop, and Badcock [55] found no significant correlation between fTCD laterality indices measured during word generation and those measured during a visuospatial landmark task.

However, not all research in this area has found evidence consistent with a statistical pattern of complementarity. To the best of our knowledge, two studies have found evidence against a statistical pattern of complementarity. Elias, Bulman-Fleming, and Guylee [56] examined the relationship 
between lateralized linguistic and prosodic processing within individuals displaying atypical laterality profiles. Elias and colleagues found the expected population-level pattern of laterality for linguistic and prosodic processing across the sample, with an overall REA for the linguistic processing task and an overall LEA for the prosodic processing task. However, in contrast to the previous findings, they observed a significant positive correlation between the two lateralized tasks suggesting a bias pattern of complementarity. The other discrepant finding in this body of research comes from a functional magnetic resonance imaging (fMRI) study examining the lateralization of face, linguistic, and visuospatial processing. Badzakova-Trajkov, Haberling, Roberts, and Corballis [57] found partial support for causal complementarity with significant negative correlations observed between LIs recorded during word generation and LIs recorded during both an emotional facial processing task and a visuospatial landmark task. An additional nonsignificant positive correlation was observed between the LIs for emotional face processing and visuospatial processing. The authors argued that these results suggest the influence of multiple lateralizing influences.

Despite the evidence in support of a left-hemisphere dominance for linguistic processing and a right-hemisphere dominance for melody recognition, the issue of complementarity has not been researched within-subjects using music processing tasks; more specifically, the relationship between melodic and linguistic processing has not yet been examined. Do these population-level biases reflect a true causal pattern at the level of the individual? The present study examined both the global, population-level pattern of lateralization for linguistic and melodic processing as well as relationship between the degrees and direction of lateralization observed for the two cognitive processes at the individual level using dichotic-listening tasks. The three patterns of complementarity provide three possible outcomes of this examination: first, if a true causal relationship exists between linguistic and melodic recognition, then a significant negative correlation between laterality scores for the two tasks should be observed, indicating clear opposite lateralization. Second, if the lateralization of these two cognitive processes is governed by an attentional or sensory system bias, then a significant positive correlation should be observed, indicating a greater bias for one hemisphere or the other overall. Third, if the mechanisms directing lateralization of these two processes are independent of one another then no significant correlation should be observed, indicating no relationship between the lateralized tasks.

\section{METHOD}

\section{Participants}

Participants in the present study were 49 (9 male and 40 female) undergraduate students from the University of Saskatchewan who participated for course credit (mean age $=19.4, S D=1.94$ ). All participants were right handed (mean score 22.35, $\mathrm{SD}=4.43$ ); handedness was assessed using the Waterloo Handedness Questionnaire (see Elias, Bryden, \& Bulman-Fleming, 1998). Right-handed participants were exclusively recruited for this study as they show a greater consistency in the lateralization of cognitive functions as compared to left-handers or mixed-handers [45, 51]. Additionally, only participants without formal music training or education were recruited. All participants in the study reported normal hearing with no history of hearing loss. The data from one participant was excluded because of a failure to follow task instructions. All participants gave written consent prior to completing the experiment, and the procedures used received approval from the Ethics Review Board of the University of Saskatchewan.

\section{Materials}

\section{Fused Dichotic Words Test (FDWT).}

Participants' language lateralization was assessed using the Fused Dichotic Words Test (FDWT) [58]. Fifteen pairs of rhymed words are presented dichotically; the rhymed words differ only on the initial phoneme sound (e.g. deer and tear). The two words fuse when presented simultaneously resulting in the perception of a single word. The rhyming word stimuli were played on CD via Windows Media Player through Sennheiser headphones (model HD-437). The rhyming word pairs were presented 16 times (eight times on each channel) for a total of 240 trials. These trials were presented in two main blocks of 120 trials each (Block A and Block B). Each of these main blocks was further divided into four blocks of 30 trials each for a total of eight blocks. Participants were asked to circle which word was heard from a list of four possible choices in the provided answer booklet.

\section{Unfamiliar Melody Recognition Task (UMRT)}

An unfamiliar melody recognition task was developed for the present study to assess lateralization for melodic processing. Kimura [18] found a significant left ear advantage for melody recognition using samples of orchestral music that differed in rhythm, timbre, and tempo. Similarly, Messerli, et al. [24] and Boucher and Bryden [26] also found overall LEAs when presenting single-instrument melodies differing in rhythm to non-musicians. As melody is typically defined as a sequential series of pitches [2] and is argued to be distinct from other musical elements such as rhythm and timbre [59] we based our task on these previous studies but simplified the stimuli, holding rhythm, timbre, and tempo constant across the melodies, altering only the pattern of pitches presented. This approach allows us to assess the lateralization of melodic pitch-sequence processing in isolation.

A stimulus set of 12 monodic melodies was created using an M-Audio Keystation Pro 88 synthesizer and Finale Allegro composition software. The stimulus melody files were produced with a sampling rate of $44.1 \mathrm{KHz}$ at a resolution of 16 bits. Each melody was seven quarter notes in length and was generated using the grand piano sound. All melodies were played in the $\mathrm{C}$ major scale, beginning on middle $\mathrm{C}(261.6 \mathrm{~Hz})$ and ending on the tonic - either $\mathrm{C} 4$ $(261.1 \mathrm{~Hz})$ or C5 $(523.3 \mathrm{~Hz})$. The note values of the melodies ranged from an octave below middle $\mathrm{C}(\mathrm{C} 3-130.8$ $\mathrm{Hz})$ to an octave above middle $\mathrm{C}(\mathrm{C} 5-523.3 \mathrm{~Hz})$. Each melody was $1000 \mathrm{msec}$ in duration, played at $240 \mathrm{bpm}$; all melodies were equalized for intensity. Each of the 12 melodies was paired dichotically with every other melody to 
create a total of 132 melody pairs. The melodies were presented using E-prime.

\section{Procedure}

Participants were tested individually. Following provision of informed consent, the participants were asked to fill out a demographics questionnaire [see 60] assessing handedness, footedness, sex, age, and the presence of vision or hearing impairments. A second questionnaire assessed musical experience. Participants then completed the FDWT and the UMRT; order of presentation of the two tasks was counterbalanced across participants. All tasks were completed in a single session approximately one hour in duration.

\section{FDWT}

The participants were seated at a table and were given a set of headphones and a response booklet to report their responses during the task. Once the task instructions had been given the participants completed a set of 30 practice trials; these trials were single-word, binaural presentations of the 30 words used to create the rhymed pairs. Participants were asked to circle which word they had heard presented in the response booklet. Following the test trials participants completed the eight blocks of test trials. The rhyming word pairs were presented with an interstimulus interval of 2.5 seconds. Participants were given a brief rest period following each block of trials; headphone position was reversed after blocks $1,3,5$, and 7 to control for channel effects.

\section{UMRT}

The participants were seated in front of a computer monitor and keyboard; they were first presented with a target melody. The melody was played five times to familiarize the participant with their specific target; the participant initiated each presentation of the target melody with a key press. Participants then completed a set of 26 practice trials. Participants were instructed to place their index and middle fingers of their right hand on the ' $y$ ' and ' $u$ ' keys, respectively. A single melody was presented binaurally following which the participant was prompted to indicate whether their target melody had been played by pressing the yes key (y) or the no key (u) on the keyboard. During sound presentation the monitor displayed a black screen; following completion of the melody presentation a response prompt was presented on the screen ("yes or no?"). The participant's response on a given trial triggered the start of the next trial. Once the practice trials were completed, the participants were prompted to press the space bar to begin the test trials.

The test trials followed the same procedure as the practice trials except a dichotic melody pair was presented in place of the binaurally presented single melody. Participants were again prompted to indicate whether their target melody was presented in either ear by pressing the yes or no key following each dichotic melody pair presentation. After completing the first block of 132 test trials, participants were assigned a new target melody, the headphones were reversed, and the task was repeated. Participants each completed two blocks of trials in total. Order of target melodies was pseudo-randomized and counterbalanced across participants.

\section{Calculation of Asymmetry Scores}

Lambda $(\lambda)$ scores were calculated for FDWT and UMRT data [see 61]:

$\lambda=\ln \left(\frac{((\mathrm{RE} \text { correct }+.005) \times(\mathrm{LE} \text { incorrect }+.005))}{((\mathrm{LE} \text { correct }+.005) \times(\mathrm{RE} \text { incorrect }+.005))}\right)$

$\mathrm{RE}$ indicates right ear responses and LE indicates left ear responses. The addition of a constant value (.005) allowed for $\lambda$ calculations if one or more of the observed values were equal to zero. This calculation provides a measure of laterality that is based on the log-odds ratio. This measure is approximately normally distributed and is independent of overall performance on the tasks. Positive $\lambda$ values reflect a right-ear performance advantage (REA) indicative of a lefthemisphere processing advantage.

\section{RESULTS}

\section{FDWT}

To assess the potential influence of block order, performance on block $\mathrm{A}$ and block $\mathrm{B}$ of the task was compared; no difference was observed, $t(47)=-.416, \mathrm{p}=$ .679. All data were collapsed for the remaining analyses. A right ear advantage (REA) was observed for 98\% (47/48) of participants for the FWDT. The overall REA for the task was significant, $t(47)=8.896, p<.001(M=.317, S D=.247)$. An analysis of overall accuracy revealed above-chance performance on the FDWT, $t(47)=85.382, p<.001$ (chance $=120, M=229.396, S D=8.878)$. See Table 1 for mean performance by ear.

\section{UMRT}

To assess a potential influence of target melody, a oneway ANOVA compared performance across all melodies; no significant difference was found, $F(12,96)=1.122, p=.355$. A $2 \times 2$ repeated measures ANOVA assessed the effects of testing block order (first and second) and ear (left and right) using percent correct performance values. The main effect of block was not significant, $F(1,47)=.633, p=.430$. Similarly, no main effect of ear was observed, $F(1,47)=$ $1.691, p=.200$. The interaction between block and ear was also not significant, $F(1,47)=1.140, p=.291$. As no significant differences between the test blocks were observed, the data were collapsed for subsequent analyses. A LEA was observed for $35 \%$ (17/48) of participants. Two participants did not elicit an ear advantage on the task; one participant was added to the REA group and the other to the LEA group for subsequent analyses. A one-sample $t$-test using the $\lambda$ scores as a measure of overall ear advantage for

Table 1. Mean Performance Percentages.

\begin{tabular}{|c|c|c|}
\hline Task & Left Ear & Right Ear \\
\hline \hline FDWT & 40.4 & 55.2 \\
\hline UMRT & 49.9 & 52.7 \\
\hline
\end{tabular}


the UMRT revealed no significant ear advantage, $t(47)=$ $1.488, p=.143(M=.271, S D=1.261)$. A one-sample t-test to assess accuracy on the task revealed that performance was above chance levels $t(47)=15.915, p<.001$ (Chance $=8 \%$ $M=51.29, S D=18.8)$. In the event that responding is random (50\% yes, $50 \%$ no) approximately half of all targetpresent trials would receive correct responses. With 22 target-present trials out of a total of 132 trials, chance performance is calculated at $8 \%$ as only $16 \%$ of trials are target-present trials.

\section{Lateral Preferences}

All participants were right-handed $(M=22.35, S D=$ 4.43). Forty-five participants showed a right-foot preference, two participants showed a left-foot preference, and one participant failed to show a preference for either foot $(M=$ $5.45, S D=3.33$ ). Handedness and footedness were found to be positively correlated, $r=.385, p=.007$. There were no significant correlations observed between performance on the FDWT and degree of handedness $(r=.147, p=.318)$ or footedness $(r=.016, p=.912)$. Significant positive correlations were observed between performance on the UMRT and degree of handedness $(r=.396, p=.005)$ as well as degree of footedness $(r=.294, p=.043)$ indicating that more strongly right-handed and right-footed participants show greater REAs (left-hemisphere lateralization) on the melody task.

\section{Correlation Between Linguistic and Melodic Processing}

We assessed the relationship between linguistic and melodic lateralization; the complementary pattern of left- hemisphere dominance (REA) for the linguistic task and right-hemisphere dominance (LEA) for the melodic recognition task was observed in 38\% (18/48) of participants. The reverse pattern of lateralization was only observed in one participant $(1 / 48=2 \%)$. Most participants displayed left-hemisphere dominance for both the linguistic and melodic recognition tasks $(29 / 48=60 \%)$. No participants displayed right-hemisphere same-side dominance for both tasks. A non-significant positive correlation between the FDWT and UMRT $\lambda$ scores was observed, $r=.259, p=.076$ (See Fig. 1).

\section{Influence of Musical Experience}

To assess the possible influence of musical experience on melody recognition performance (operationalized as the number of hours spent listening to music per week), we performed a regression analysis using the overall task accuracy as the independent variable and the number of hours spent listening to music per week as the dependent variable. There was no significant relationship between musical experience and overall UMRT task accuracy observed $(r=.107, b=.003, p=.474)$. This suggests that participants who spend more time listening to music do not tend to show greater accuracy on the UMRT than those participants who do not listen to music as frequently. Similarly, we assessed the relationship between the observed $\lambda$ values for the UMRT task and musical experience. Again, no significant relationship was observed $(r=.016, b=.003$, $p=.915)$. This result suggests that participants who listen to music more frequently are not more likely to show greater bias scores (ear-advantages) on the UMRT.

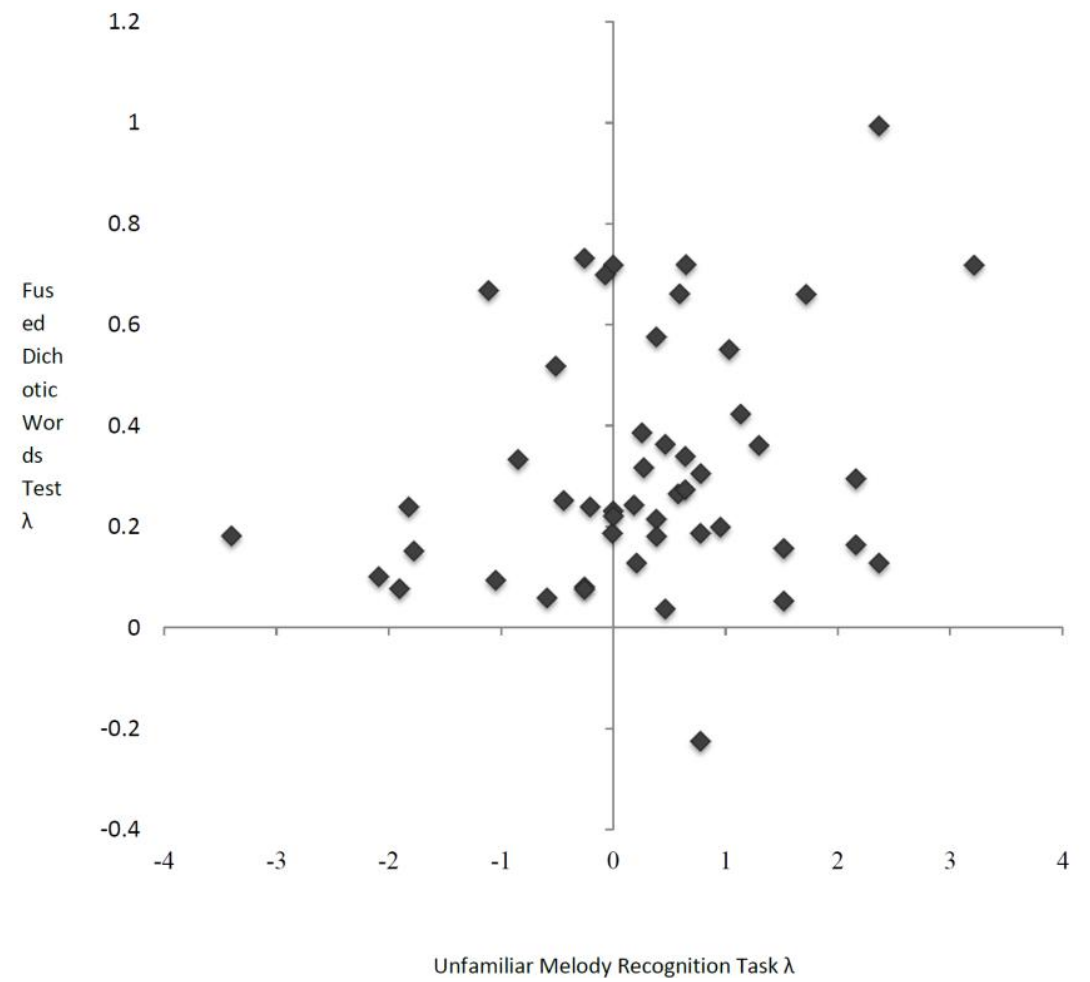

Fig. (1). Individual $\lambda$ scores on the Fused Dichotic Words Test (FDWT) versus $\lambda$ scores on the Unfamiliar Melody Recognition Task (UMRT) for all participants. Positive values indicate a right ear advantage (REA). 


\section{DISCUSSION}

We examined the pattern of complementarity observed between linguistic processing (FDWT) and unfamiliar melody recognition (UMRT). We observed the expected REA in the linguistic task, but failed to observe a significant LEA for unfamiliar melody recognition. The number of hours spent listening to music did not influence either performance or degree of lateralization on the melody recognition task. Handedness and footedness were both found to be positively correlated with degree of lateralization for melody recognition. Finally, no relationship was observed between degree of laterality for the linguistic and melodic recognition tasks.

The overall REA observed for the linguistic processing task is consistent with the previous findings of a lefthemisphere dominance for language [32,39]. The failure to find a significant LEA for the melody recognition task was unexpected, but can be explained by a shift in processing strategy for the task. Despite the evidence from studies demonstrating that melody recognition is more efficient when pitch information is presented in isolation from rhythm information [62, 63], there is evidence suggesting that melodic processing is inherently rhythmical [64]. Kidd, Boltz, and Jones [65] found that melodies with different pitch sequences were judged as being the same when they shared the same rhythmic structure. This suggests that both pitch and rhythm information may be perceived and processed as a single dimension in melody recognition. In fact, responses from participants following task completion in the present study frequently indicated that they did not hear the melody tokens as true melodies, rather they were perceived as a non-unified string of notes. The absence of rhythmic variation was often cited as the cause of this perception.

The similarity of rhythm across all the tokens in the UMRT, in addition to the limited pitch range and the similar starting note across tokens, may have biased participants to view the melodies as highly similar. Together these conditions may have resulted in participants switching from a global, contour-based strategy for melodic processing to a more local, interval-based strategy. There is evidence to suggest that interval and contour information are processed with opposite hemispheres (left and right, respectively) [15, 66-68]. Peretz, et al. [15] demonstrated that a shift from a contour-based holistic processing strategy (LEA) to an interval-based analytic (REA) strategy could be induced by prompting participants to focus on specific notes or intervals within a presented melody. Such a shift in processing would result in increased reliance on left-hemisphere analytic processes, thus potentially explaining our failure to find a LEA for the UMRT.

A non-significant positive correlation between the $\lambda$ values for the FDWT and the UMRT was observed. The failure to find a significant negative relationship suggests that participants showing a REA for the linguistic task are not more likely to show an LEA on the melody recognition task, as would be predicted by the Modal Model. This finding is not surprising, given the failure to elicit the expected LEA with the melody recognition task. As such, the relationship between the linguistic and melodic task laterality scores is not informative regarding the nature of complementary lateralization of the two functions; however, the positive direction of the correlation, especially given the REA observed for both tasks, does provide some evidence in support of a shared processing network for the processing of linguistic and elements of musical stimuli.

Neuropsychological evidence for dissociation between the mechanisms governing music and language processing is compelling [19]. For example, Peretz [69] provided a description of patient G.L. who, following bilateral temporal lobe damage, demonstrated a significant impairment in pitch tone interpretation in the absence of language processing difficulties. Conversely, Poeppel [70] presents a case where bilateral temporal lobe damage resulted in total impairment in understanding spoken language while preserving the ability to process and identify musical or other environmental sounds. However, neuroimaging studies have provided evidence suggesting that significant overlap exists in the processing networks for music and language [4, 71]. For example, Brown, Martinez, and Parsons [3] had participants complete sentence and melody fragments with spontaneous, improvised responses. Using positron emission tomography (PET), the authors found bilateral activation in superior temporal and inferior frontal regions for both the music and language generation tasks. In fact, the real difference between music and language processing did not seem to lie in the processing locations, rather more in the lateralization of the functions, with greater left hemisphere activation observed for language processing. They argued that these results provide evidence for a shared role in the generation of complex sounds.

Zatorre and Belin [72] suggest that the left and right hemispheres are not specialized for language and music, respectively, but rather are specialized for specific aspects of sound processing, with the left hemisphere specialized for rapid temporal processing and the right hemisphere specialized for general pitch processing. According to this view, it is possible that participants resorting to an interval processing approach to the melody recognition task may have engaged left-hemisphere temporal processing mechanisms to process the temporal order of the relative pitches in the presented melodies. The observed positive correlation between the linguistic and melodic processing tasks in our study may indicate a shared processing mechanism, as effective processing of linguistic stimuli requires rapid temporal sequencing.

Alternatively, Patel [73] argues that music and language each have a domain-specific representational network where the knowledge systems for the two processes are thought to be independent [74]. This can be thought of as a rule-book for the syntax and structural rules governing language and music composition. Patel also suggests that music and language each have a resource network used in the processing and integration of information during processing, and that these networks overlap resulting in shared processing. In essence, the resource networks are a sorting and organizing system allowing for rapid selection and associative activation of information during online processing. According to this view, it is possible that both the linguistic and melody recognition tasks in our study 
showed similar reliance on these shared resources for the rapid sequencing and processing of the structural information of both the words and melodies presented. To differentiate between these two potential explanations, further research is needed.

As our ability to examine the relationship between lateralization of linguistic and melodic processing was precluded by the failure to elicit the expected LEA for melodic processing, conclusions about the nature of the complementary relationship between language and melodic processing cannot be drawn. Additional examination of this relationship is needed using tasks that more accurately capture the typical population-level pattern of lateralization. Further study of the relationship between left-lateralized linguistic processing and right-lateralized melodic processing would benefit from focusing more specifically on melodic contour information or including temporal information in the melodies presented in an attempt to reduce reliance on left-hemisphere processing strategies.

This research was supported by a Canada Graduate Scholarship from the Natural Sciences and Engineering Research Council of Canada to V. Harms, and by a grant from the Natural Sciences and Engineering Research Council of Canada to L. J. Elias.The authors wish to thank two anonymous reviewers for their helpful feedback on an earlier version of this manuscript.

\section{REFERENCES}

[1] Nettl B. An ethnomusicologist contemplates universals in musical sound and musical culture. In: Wallin NL, Merker B, Brown S, Eds. The Origins of Music, Cambridge, MA: MIT Press 2000; pp. 463-79.

[2] Patel AD. Music, Language, and the Brain. Toronto: Oxford University Press 2008.

[3] Brown S, Martinez MJ, Parsons LM. Music and language side by side in the brain: a pet study of the generation of melodies and sentences. Eur J Neurosci 2006; 23: 2791-803.

[4] Levitin DJ, Menon V. Musical structure is processed in "Language" areas of the brain: a possible role for Brodmann Area 47 in temporal coherence. Neuroimage 2003; 20: 2142-52.

[5] Schön D, Gordon, RL, Besson M. Musical and linguistic processing in song perception. Ann NY Acad Sci 2005; 1060: 7181.

[6] Peretz I, Zatorre RJ. Brain organization for music processing. Ann Rev Psychol 2005; 56: 89-114.

[7] Bever TG, Chiarello RJ. Cerebral dominance in musicians and nonmusicians. Science 1974; 185: 537-39.

[8] Falk D. Hominid brain evolution and the origins of music. In: Wallin NL, Merker B, Brown S, Eds. The origins of music. Cambridge, MA: MIT Press 2000; pp. 197-216.

[9] Jerison $\mathrm{H}$. Paleoneurology and the biology of music. In: Wallin NL, Merker B, Brown S, Eds. The origins of music. Cambridge, MA: MIT Press 2000; pp. 177-96.

[10] Finlay BL, Darlington RB. Linked regularities in the development and evolution of mammalian brains. Science 1995; 268: 1578-84.

[11] Sakai K, Hikosaka O, Miyauchi S, et al. Neural representation of a rhythm depends on its interval ratio. J Neurosci 1999; 19: 10074-81

[12] Ayotte J, Peretz I, Rousseau I, et al. Patterns of music agnosia associated with middle cerebral artifacts. Brain 2000; 123: 192638.

[13] Platel H, Price C, Baron JC, et al. The structural components of music perception. a functional anatomical study. Brain 1997: 120: 229-43.

[14] Peretz I. Processing of local and global music information by unilateral brain-damaged patients. Brain 1990; 113: 1185-205.
[15] Peretz I, Morais J, Bertelson P. Shifting ear differences in melody recognition through strategy inducement. Brain Cognit 1987; 6: 202-15.

[16] Liégeois-Chauvel C, Peretz I, Babai M, et al. Contribution of different cortical areas in the temporal lobes to music processing. Brain 1998; 121: 1853-67.

[17] Koelsch S, Gunter TC, Wittforth M, et al. Interaction between syntax processing in language and music: an erp study of music perception. J Cognit Neurosci 2005; 17: 1565-77.

[18] Kimura D. Left-right differences in the perception of melodies. Q J Exp Psychol 1964; 16: 355-8.

[19] Marin OSM, Perry DW. Neurological aspects of music perception and performance. in: Deutsch D, ed. the psychology of music. San Diego, CA: Academic Press 1999; pp. 653-724.

[20] Milner B. Laterality effects in audition. In: Mountcastle V, Ed. Interhemispheric relations and cerebral dominance. Baltimore, MD: Johns Hopkins Press 1962; pp. 177-95.

[21] Samson S. Zatorre RJ. Recognition memory for text and melody of songs after unilateral temporal lobe lesion: evidence for dual encoding. J Exp Psychol Learn 1991; 17: 793-804.

[22] Samson S. Zatorre RJ. Melodic and harmonic discrimination following unilateral cerebral excision. Brain Cognit 1988; 7: 34860 .

[23] Steinke WR, Cuddy LL, Jakobson LS. Dissociations among functional subsystems governing melody recognition after righthemisphere damage. Cognit Neuropsych 2001; 18: 411-37.

[24] Messerli P, Pegna A, Sordet N. Hemispheric dominance for melody recognition in musicians and non-musicians. Neuropsychologia 1995; 33: 395-405.

[25] Zatorre RJ. Recognition of dichotic melodies by musicians and nonmusicians. Neuropsychologia 1979; 17: 607-17.

[26] Boucher R, Bryden MP. Laterality effects in the processing of melody and timbre. Neuropsychologica 1997; 35: 1467-73.

[27] Peretz I, Gosselin N, Belin P, et al. Music lexical networks: the cortical organization of music recognition. Ann NY Acad Sci 2009; 1169: 256-65.

[28] Platel H, Baron JC, Desgranges B, et al. Semantic and episodic memory of music are subserved by distinct neural networks. Neuroimage 2003; 20: 244-56.

[29] Patterson RD, Uppenkamp S, Johnsrude IS, et al. The processing of temporal pitch and melody information in auditory cortex. Neuron 2002; 36: 767-76.

[30] Belin P, Zatorre RJ, Ahad P. Human temporal-lobe response to vocal sounds. Cognit Brain Res 2002; 13: 17-26.

[31] Grimshaw GM. Integration and interference in the cerebral hemispheres: relations with hemispheric specialization. Brain Cognit 1998: 36: 108-27.

[32] Ley RG, Bryden M. A dissociation of right and left hemisphere effects for recognizing emotional tone and verbal content. Brain Cognit 1982; 1: 3-9.

[33] Poeppel D. The analysis of speech in different temporal integration windows: cerebral lateralisation as 'Asymmetric Sampling In Time'. Speech Commun 2003; 41: 245-55.

[34] Lindell AK. In your right mind: right hemisphere contributions to language processing and production. Neuropsychol Rev 2006; 16: 131-48.

[35] Broca P. Remarques Sur Le Sie 'Ge De La Faculte' De La Parole Articule'E, Suivies D'une Observation D'aphe'Mie (Perte De Parole). B Soc Anat 1861; 36: 330-57.

[36] Friederici $\mathrm{AD}$, Alter $\mathrm{K}$. Lateralisation of auditory language functions: A dynamic dual pathway model. Brain Lang 2004; 89: 267-76.

[37] Geschwind N. Specializations of the human brain. Sci Am 1979; $241 ; 180-99$

[38] Hecaen H, DeAgostini M, Monzon-Montes A. Cerebral organization in left-handers. Brain Lang 1981; 12: 261-84.

[39] Kimura D. Cerebral dominance and the perception of verbal stimuli. Can J Psychol 1961; 15: 166-71.

[40] Bryden MP. Choosing sides: The left and right of the normal brain. Can Psychol 1990; 31: 297-309.

[41] Corballis MC. The Lopsided Ape. New York, NY: Oxford University Press 1991.

[42] Sperry RW. Some effects of disconnecting the cerebral hemispheres. Science 1982; 217: 1223-7.

[43] Bradshaw JL. Asymmetries in preparation for action. Trends Cogn Sci 2001; 5: 184-5. 
[44] Levy J. The mammalian brain and the adaptive advantage of cerebral asymmetry. Ann NYAcad Sci 1977; 299: 264-72.

[45] Hellige JB. Hemispheric asymmetry: what's right and what's left. Cambridge, MA: Harvard University Press 1993.

[46] Bryden MP, Hecaen H, DeAgostini M. Patterns of cerebral organization. Brain Lang 1983; 20: 249-62.

[47] Efron R, Koss B, Yund EW. Central Auditory procession. iv. ear dominance - spatial and temporal complexity. Brain Lang 1983; 19: 264-82.

[48] Kinsbourne M. The mechanisms of hemispheric control of the lateral gradient of attention. In: Rabbit PM, Doric S, Eds. Attention And Performance. London, UK: Academic Press 1975; pp. 81-97.

[49] Sidtis JJ. Predicting brain organization from dichotic listening performance: cortical and subcortical functional asymmetries contribute to perceptual asymmetries. Brain Lang 1982; 17: 287300.

[50] Teng E. Dichotic ear difference is a poor index for the functional asymmetry between the cerebral hemispheres. Neuropsychologia 1981; 19: 235-40.

[51] Bryden MP. Laterality: functional asymmetry in the intact brain. New York, NY: Academic Press 1982.

[52] Saxby L, Bryden MP. Left-ear superiority in children for processing auditory emotional material. Dev Psychol 1984; 20: 7280.

[53] McNeely HE. Parlow SE. Complementarity of linguistic and prosodic processes in the intact brain. Brain Lang 2001; 79: 47381.

[54] Whitehouse AJO, Bishop DVM. Hemispheric division of function is the result of individual probabilistic biases. Neuropsychologia 2009; 47: 1938-43.

[55] Rosch RE, Bishop DVM, Badcock NA. Lateralised visual attention is unrelated to language lateralisation, and not influenced by task difficulty - a functional transcranial doppler study. Neuropsychologia 2012; 50: 810-15.

[56] Elias LJ, Bulman-Fleming MB, Guylee MJ. Complementarity of cerebral function among individuals with atypical laterality profiles. Brain Cognit 1999; 40: 112-5.

[57] Badzakova-Trajkov G, Haberling IS, Roberts RP, et al. Cerebral asymmetries: complementary and independent processes. PLoS ONE 2010; 5: e9682.

[58] Wexler BE, Halwes T. Increasing the power of dichotic methods: the fused rhymed words test. Neuropsychologia 1983; 21: 59-66.
[59] Limb CJ. Structural and functional neural correlates of music perception. Anat Rec 2006; 288: 435-46.

[60] Elias LJ, Bryden MP, Bulman-Fleming MB. Footedness is a better predictor than is handedness of emotional lateralization. Neuropsychologia 1998; 36: 37-43.

[61] Bryden MP, Sprott DA. Statistical determination of degree of laterality. Neuropsychologica 1981; 19: 571-81

[62] Hebert S, Peretz I. Recognition of music in long-term memory: are melodic and temporal patterns equal partners? Mem Cognit 1997; 25: 518-33.

[63] White B. Recognition of distorted melodies. Am J Psychol 1960; 7: 253-8.

[64] Jones MR, Boltz M. Dynamic attending and responses to time Psychol Rev 1989; 96: 459-91.

[65] Kidd G, Bolts M, Jones MR. Some effects of rhythmic context on melody recognition. Am J Psychol 1984; 97: 153-73.

[66] Peretz I, Babai M. The role of contour and intervals in the recognition of melody parts: evidence from cerebral asymmetries in musicians. Neuropsychologia 1992; 30: 277-92.

[67] Lee Y, Janata P, Frost C, et al. Investigation of melodic contour processing in the brain using multivariate pattern-based fMRI Neuroimage 2011; 57: 293-300.

[68] Stewart L, Overath T, Warren JD, et al. fMRI evidence for a cortical hierarchy of pitch pattern processing. PLoS ONE 2008; 3: e1470.

[69] Peretz I. Auditory agnosia: a functional analysis. In: McAdams S, Bigand E, Ed. Thinking in sound: the cognitive psychology of human audition. Oxford: Clarendon Press 1993; pp. 199-230.

[70] Poeppel D. Pure word deafness and the bilateral processing of speech code. Cognitive Sci 2001; 21: 679-93.

[71] Hickock G, Buchsbaum B, Humphries C, et al. Auditory-motor integration revealed by fMRI: speech, music, and working memory in area SPT. J Cognit Neurosci 2003; 15: 673-82.

[72] Zatorre RJ, Belin P. Spectral and temporal processing in human auditory cortex. Cereb Cortex 2001; 11: 946-53.

[73] Patel AD. Language, music, syntax, and the brain. Nat Neurosci 2003; 6: 674-81.

[74] Federenko E, Patel A, Casasanto D, et al. Structural integration in language and music: evidence for a shared system. Mem Cognit 2009; 37: 1-9.

Received: November 05, 2013

Revised: December 16, 2013

Accepted: December 16, 2013

(c) Harms et al.; Licensee Bentham Open.

This is an open access article licensed under the terms of the Creative Commons Attribution Non-Commercial License (http://creativecommons.org/licenses/by-nc/3.0/) which permits unrestricted, non-commercial use, distribution and reproduction in any medium, provided the work is properly cited.p 